\title{
O uso do tablet para a representação de conceitos de genética: proposta e análise com base na Teoria da Atividade
}

\author{
Rosiney Rocha Almeida -UNICSUL - rrrosyy@yahoo.com.br \\ Carlos Fernando Araújo Jr - UNICSUL - carlos.araujo@cruzeirodosul.edu.br \\ Meire Pereira França - UNICSUL - meirepf@ig.com.br
}

\begin{abstract}
Resumo: Este artigo analisa se o uso do tablet pode colaborar com a aquisição da competência de representação de conhecimentos de genética sob a forma de conceitos. Com base nos fundamentos teórico-metodológicos da Teoria da Atividade (TA), foi desenvolvida e analisada uma atividade de representação do conhecimento, que consistiu, dentre outras ações, na elaboração de três mapas mentais digitais, para cada aluno, relacionados a conteúdos de genética. Participaram deste estudo 34 alunos da $3^{\text {a }}$ série do Ensino Médio, do Colégio Cruzeiro do Sul, localizado na cidade de São Paulo; duas professoras pesquisadoras no âmbito da aprendizagem móvel; e dois técnicos em Informática. Os resultados indicaram uma evolução no número de conceitos adequados representados pelos alunos no decorrer da atividade realizada. Observou-se que o tablet, quando utilizado sob a perspectiva da TA, pode colaborar com a representação de conhecimentos de conteúdos de genética, sob a forma de conceitos.
\end{abstract}

Palavras-chave: mobile-learning. tablet no ensino. teoria da atividade.

\section{The use of tablet to representation of genetics concepts: purporse and analysis based on Theory of Activity}

\begin{abstract}
This paper analyzes if the use of tablet can contribute with the acquisition of the competence of representation of knowledge of genetics in the way of concept. Based on Theory of Activity (TA), a activity of representation of knowledge was developed and it was analyzed. This activity was based on three mental digital maps related to genetics and it was applied for each student, 34 students of third grade in High School level, from Colégio Cruzeiro do Sul, located in São Paulo; two teachers who research mobile learning; and two information technology technicians. The results indicated the evolution of the number of adequate represented concepts by the students. Furthermore, it was observed that the tablet, when is used and based in adequate theory like TA, can contribute with the representation of knowledge of genetics in the way of concept.
\end{abstract}

Keywords: mobile-learning. tablet in education. theory of activity.

\section{INTRODUÇÃO}

De acordo com Higuchi (2011), a constante inserção de tecnologias móveis no processo de ensino e aprendizagem tem favorecido o surgimento de pesquisas com interesse em investigar de que forma alguns dispositivos, exemplares dessas 
tecnologias, como smartphones, tablets, podem contribuir no processo mobile learning (m-learning). Existem muitos conceitos ou definições sobre m-learning que foram recentemente sintetizados em Araújo Jr e Silveira (2012). Consideramos como uma conceituação abrangente a dada por Crompton (2013): m-learning é o aprendizado realizado por múltiplos contextos, com interações em conteúdos e sociais, usando dispositivos eletrônicos de uso pessoal.

Araujo Jr e Silveira (2012) e colaboradores realizaram um estudo recente e aprofundado envolvendo diversos usos (atividades) do tablet nas áreas de Ciências e Matemática, mas especificamente em Matemática, Física e Biologia, tendo como base do desenvolvimento das atividades a TA. Contudo, acreditamos, conforme apontam Viera e Santarosa (2013), que há ainda uma reduzida expressão de investigações que analisam os impactos e as possibilidades pedagógicas no uso desses dispositivos. Desse modo, a necessidade de construção de novos cenários educativos abertos à inovação e à interação são fatores preponderantes para a realização da presente pesquisa, justificando-a, pois.

Nesse sentido, tendo em vista que uma das competências previstas para o Ensino de Biologia nos Parâmetros Curriculares Nacionais é a de "Representação de dados publicados em livros, revistas, jornais ou documentos oficiais, na forma de gráficos, tabelas, esquemas e interpretá-los criticamente" (BRASIL, 2002, p. 36), este estudo tem como objetivo analisar se o uso do tablet pode colaborar com a aquisição da competência de representação de conhecimentos de conteúdos de genética sob a forma de conceitos.

\section{O TABLET NO PROCESSO DE ENSINO E APRENDIZAGEM}

Oliveira et al. (2012) declara que a lista de material escolar no início do ano letivo, em algumas escolas, está sendo substituída por um único item. No lugar da mochila cheia de livros, cadernos e lápis, os tablets começam a fazer parte do ambiente escolar. Nesse contexto, o autor afirma que é possível ao aluno pesquisar, digitar, ler e interagir com aplicativos, dinamizando a aula.

Para o autor, é inevitável a inclusão desse recurso, em todo o sistema de ensino, pois algumas escolas particulares já as utilizam. As escolas públicas, por sua vez, já começam a ter um forte incentivo do governo e investimentos para a compra desse recurso e de sua utilização em sala de aula. O uso do tablet propicia a interação, a partilha de significados e a colaboração, viabilizando uma educação contextualizada cujos conteúdos estejam adequados às necessidades da sociedade contemporânea.

Os professores ao adotarem, em sua prática, esses recursos tecnológicos, oferecem possibilidades de pesquisa, leitura e comunicação próximas dos alunos e dos ambientes digitais que eles frequentam, podendo motivá-los ainda mais à aprendizagem.

\section{A TEORIA DA ATIVIDADE COMO APORTE TEÓRICO METODOLÓGICO DAS PESQUISAS EM M-LEARNING}

Nesse sentido, Sharples et al (2005), um dos pioneiros na tentativa de estabelecer um diálogo conducente com uma teoria para o m-learning, sugere a Teoria da Atividade (TA) como teoria apropriada para sustentar um modelo de aprendizagem baseado em tecnologias móveis, porque analisa a aprendizagem como um sistema de atividade histórico-cultural mediado por ferramentas ou recursos que apoiam os alunos nos objetivos de transformação dos seus conhecimentos e competências. 
Leontiev, um dos continuadores dos trabalhos de Vygotsky (1978), é , caracteriza o conceito de atividade:

[...] Por atividade, designamos os processos psicologicamente caracterizados por aquilo a que o processo, como um todo, se dirige (seu objeto), coincidindo sempre com o objetivo que estimula o sujeito a executar esta atividade, isto é, o motivo (LEONTIEV, 1988, p. 68).

Nesse campo, Kaptelin e Nardi (1997) organizaram cinco princípios da TA. Neste estudo, porém, baseamo-nos nos princípios da Mediação e da Estrutura Hierárquica da Atividade.

O conceito de Mediação foi introduzido por Vygotsky (1978), na Primeira geração da TA, e possui a noção de mediação entre o sujeito e objeto como principal característica desta geração. Kaptelin \& Nardi (1997) enumeram o princípio da mediação como um dos princípios desta teoria e dizem que toda atividade é mediada por artefatos, sejam eles materiais e/ou imaginários (símbolos, signos, normas, ferramentas etc.).

O tablet, concebido como um produto sócio-histórico-cultural, vem sendo apropriado na educação como meio para aprender, ensinar, e busca-se, nessa teoria, fundamentação para nosso estudo.

Ademais, Engeström (1987), na Segunda geração da TA, considera a mediação para além da questão sujeito-artefato-objeto e admite também os elementos sociais de mediação como a comunidade envolvida na atividade, que no contexto de uma atividade pedagógica, como é o caso deste estudo, pode ser o professor e os demais alunos envolvidos na atividade.

A atividade humana é organizada por três níveis hierárquicos que se complementam: a Atividade, a Ação e a Operação.

[...] Ao levar em consideração os objetivos para os quais cada nível é orientado, temos que a atividade é orientada a motivos, a ação orientada a metas e a operação orientada a condições de realização. Assim, a atividade do professor é um conjunto de ações intencionais, conscientes, dirigidas para um fim específico (BASSO, 1998, p. 4).

As ações, componentes fundamentais da atividade, são implementadas mediante operações. Cada ação inclui diferentes operações que dependem das condições de execução da ação. As operações, como já mencionado, são as formas ou as condições de realização das ações.

Uma visão mais detalhada sobre os diversos usos da TA no contexto do $m$ learning pode ser obtida em Araújo Jr e Silveira (2012) e, mais especificamente sobre TA, em Almeida e Araújo Jr (2012).

\section{MATERIAL E MÉTODOS}

Este estudo consiste em uma pesquisa qualitativa e baseia-se nos fundamentos teórico-metodológicos da TA. Em um período de dois meses e meio foi desenvolvida e analisada uma Atividade de representação de conhecimentos relacionados a conteúdos de genética.

\subsection{Participantes da pesquisa}


Participaram deste estudo 34 alunos da $3^{\text {a }}$ série do Ensino Médio, do Colégio Cruzeiro do Sul, localizado na cidade de São Paulo; dois Técnicos em Informática, os quais deram suporte técnico durante a atividade realizada; e duas professoras de Biologia e pesquisadoras do Projeto de Pesquisa Este projeto consiste em uma pesquisa temática longitudinal com o objetivo de estudar e analisar os usos e impactos dos tablets no ensino fundamental e médio na área de ensino de Ciências e Matemática (ARAÚJO JR e SILVEIRA, 2012).

\subsection{Viabilização da pesquisa} providências:

Para viabilizar a realização do estudo, foram tomadas as seguintes

4.2.1 Distribuição dos alunos em grupos: Os alunos foram organizados em nove grupos relacionados aos contéudos: Grupo 1 - O código genético; Grupo 2 - Mutações gênicas; Grupo 3 - Reprodução celular; Grupo 4 - Primeira Lei de Mendel; Grupo 5 Alelos múltiplos; Grupo 6 - Herança e sexo; Grupo 7 - Segunda Lei de Mendel; Grupo 8 - Interação gênica; e Grupo 9 - Engenharia genética. Os alunos foram distribuídos aleatoriamente entre os nove grupos, com média de quatro alunos por grupo.

4.2.2 Distribuição dos tablets: foi distribuído, para cada aluno, um tablet da marca Positivo YPY 10 Android, 4.0 com acesso à internet, e cada aluno trabalhou com ele individualmente, durante as aulas de Biologia do Colégio Cruzeiro do Sul. No final de cada aula, os tablets eram recolhidos, pois pertencem ao acervo do citado projeto.

4.2.3 Criação da Disciplina no Blackboard: foi criada, no Blackboard, a Disciplina Tablets no Ensino de genética. Em seguida, os alunos foram matriculados na disciplina nos seus respectivos grupos. O ambiente Blackboard é um ambiente virtual de aprendizagem utilizado em várias instituições de ensino superior, no Brasil e no exterior, e mais recentemente em escolas de nível fundamental e médio.

4.2.4. Mapeamento mental como técnica de coleta de dados: na ocasião da pesquisa, diante da não disponibilidade de um programa para elaborar mapas conceituais, compatível com a plataforma Android (adotada neste estudo), optou-se por adotar o mapeamento mental (BUZAN, 2005) para a representação dos conhecimentos obtidos sob a forma de conceitos pelos alunos. O mapeamento mental foi utilizado como técnica de coleta de dados a fim de capturar e arquivar a número dos conceitos relacionados aos conteúdos de genética, aqui investigados, que os alunos conseguiram representar ao longo do estudo. Para elaboração dos Mapas Mentais, optou-se pelo aplicativo SchematicMind(ref).

\subsection{Realização da Atividade}

As ações principais dos alunos, durante a Atividade (LEONTIEV, 1978) de representação do conhecimento com o uso do tablet, consistiram na elaboração de três mapas mentais digitais: mapa mental 1, elaborado a partir do conhecimento prévio do aluno; mapa mental 2, elaborado a partir da interação com os recursos pesquisados via tablet (artefato mediador) e; finalmente, o mapa mental 3, elaborado pelos alunos a partir da mediação das professoras e colegas (mediadores sociais). A atividade foi desenvolvida ao longo de sete encontros descritos a seguir. 
$1^{0}$ Encontro - Apresentação geral dos conteúdos de genética: foi realizada uma apresentação dos conteúdos de genética investigados com o objetivo de oferecer aos alunos uma visão geral da temática proposta, bem como dos assuntos a ela relacionados.

$2^{\circ}$ Encontro - Elaboração do primeiro mapa mental digital: no segundo encontro a atividade foi dividida nas seguintes etapas; a) orientações sobre a representação dos conceitos através do mapeamento mental: foram passadas orientações aos alunos acerca do conceito, aplicabilidade, importância da utilização de Mapas Mentais para o ensino e a aprendizagem. Em seguida, foi apresentado o SchematicMind, aplicativo adotado no estudo; b) elaboração do primeiro mapa mental digital: cada aluno elaborou individualmente seu primeiro mapa mental. Essa elaboração teve como conceito raiz o referente ao tema do grupo de cada aluno. Os alunos foram orientados a elaborar o primeiro Mapa sem consultar livros, apostilas ou recursos da internet; apenas com base no conhecimento prévio, ou seja, no seu conhecimento construído no cotidiano sobre o assunto; c) Compartilhamento do primeiro mapa no fórum de discussões do Blackboard: finalizada a elaboração do Primeiro Mapa Mental Digital, cada aluno "printou" o seu Mapa e compartilhou no Fórum de discussões referente ao seu grupo. A professora apresentou um feedback aos alunos em relação ao primeiro mapa.

$3^{\circ}$ Encontro - Elaboração do segundo mapa mental. Neste encontro a atividade teve como objetivos específicos: a) a busca por novos conhecimentos na web através do tablet: tomando como base o Princípio da Mediação da TA, os alunos foram orientados a, individualmente, utilizando o tablet, realizarem buscas por recurso(s) relacionado(s) ao tema do seu grupo e que lhes trouxessem novos conhecimentos na $w e b$. Foram realizadas algumas orientações e sugestões de busca pelas professoras, como palavras-chave e orientações em relação à procura por esses recursos. Mesmo tendo sido oferecidas algumas sugestões de busca pelas professoras, procurou-se respeitar a maneira criadora com que cada aluno lida com o dispositivo e com os recursos que eles poderiam encontrar na web; b) Elaboração do segundo mapa mental Digital: após identificarem os conceitos relacionados ao tema presentes nos recursos pesquisados, os alunos foram orientados a procederam, de forma individual, à representação dos conceitos encontrados, através da elaboração do Segundo Mapa Mental digital; c) Compartilhamento do segundo mapa mental e do(s) recursos explorado(s) no fórum de discussões do Blackboard: finalizada a elaboração do Mapa, os alunos compartilharam no fórum de discussões de seus respectivos grupos o Segundo Mapa digital elaborado, bem como o(s) "links" com o endereço do(s) recurso(s) visitado(s) e; d) Exploração dos Mapas Mentais e dos recursos compartilhados pelos colegas: depois do compartilhamento do Segundo Mapa Mental digital e do(s) $\operatorname{link}(s)$ do(s) recurso(s) pesquisado(s), os alunos foram estimulados pelas professoras a explorarem os Mapas Mentais e os recursos partilhados pelos colegas do seu grupo a fim de promover uma troca dinâmica de conhecimentos entre eles.

$4^{\circ} 5^{\circ}$ e $6^{\circ}$ Encontros - Explicação dos mapas pelos alunos e mediação do processo de ensino e aprendizagem

Durante o $4^{\circ}, 5^{\circ}$ e $6^{\circ}$ encontros, procedeu-se à explicação individual do segundo mapa mental acompanhada da mediação do processo de ensino e aprendizagem pelas professoras e os demais alunos envolvidas na pesquisa. Esse foi o momento da pesquisa em que cada aluno explicou para a turma o seu mapa e apresentou o(s) recurso 
(s) explorado(s). Nesse momento da pesquisa, a mediação feita pelas professoras no processo de ensino e aprendizagem ocorreu de forma mais expressiva. A mediação feita pelas professoras se deu, principalmente, de forma a questionar a ausência de um conceito identificado no recurso explorado ou o posicionamento de conceitos que necessitam ser reposicionados no mapa. Nessa etapa de explicação dos mapas conceituais, o erro se configurou como indicador diagnóstico a ser interpretado para orientar ações na elaboração da terceira versão do mapa.

A análise dos três mapas foi realizada pelas professoras pesquisadoras tendo como critério de análise o número de conceitos adequados representado em cada mapa.

Neste segundo mapa, em específico, após ter sido dada a oportunidade aos alunos de se expressarem, explicando o caminho percorrido para chegarem à sua elaboração, foi realizada a sua análise pelas professoras em um momento posterior às explicações, tendo como base os mapas, as declarações dos alunos e as mediações realizadas durante a apresentação. A análise se deu com base na exploração dos recursos utilizados pelos alunos para a elaboração do Mapa, seguida da busca de correspondência dos conceitos identificados nos recursos com os conceitos representados nos mapas mentais. Em seguida, foi contado o número de conceitos adequados representados no mapa. Com base na identificação pelas professoras dos conceitos identificados nos recursos que o aluno ainda não compreendia, foi dado um retorno específico para cada aluno envolvido na pesquisa. O resultado da análise de cada mapa foi depositado no banco de discussões do Blackboard de cada grupo.

\section{$7^{\circ}$ Encontro - Elaboração do terceiro mapa mental digital e compartilhamento}

O sétimo encontro teve realizou-se em duas etapas: a) elaboração do terceiro mapa mental digital: com base na possibilidade de arquivo dos mapas no tablet para posterior edição, cada aluno retomou a edição anterior do seu mapa e procedeu à elaboração da Terceira versão do mapa conceitual digital, agora baseados nas considerações apresentadas durante a explicação pelos demais alunos e professoras e no resultado da análise feita pelas professoras, compartilhada no fórum de discussões do Blackboard $e$; b) compartilhamento do terceiro mapa mental no fórum de discussões do Blackboard: o terceiro Mapa conceitual Digital foi compartilhado no Blackboard por cada aluno, depois de feitas as adequações. Ao final dessa atividade, os conhecimentos adquiridos ( $3^{\circ}$ Mapa Mental) foram avaliados comparativamente com os conhecimentos prévios, para analisar se houve a ocorrência de "evolução conceitual" da amostra.

\section{RESULTADOS}

Nas Figuras 1, 2 e 3 apresentamos a síntese da quantidade de conceitos representados pelos alunos em intervalos de [0-5], [6-10], [11-15] e [16-20] e maior que 20. Nossa pressuposição teórica, de uma forma mais abrangente, de que a representação do conhecimento, por meio de conceitos e suas conexões, realizada por meio de atividades de aprendizagem, baseada na TA utilizando os tablets e o aplicativo SchematicMind, desenvolvidas de forma individual e coletiva poderiam contribuir para competência dos alunos em representação de conhecimentos na forma conceitos. Na Figura 1, apresentamos os dados relativos à análise do primeiro mapa digital elaborado pelos alunos a partir do seu conhecimento prévio acerca dos conteúdos de genética a serem pesquisados, 27 dos 34 alunos que participaram da pesquisa representaram em seus mapas até 10 conceitos adequados, sendo que 11 destes representaram no máximo 
5 conceitos adequados em seu mapa. Apenas 7 alunos representaram um número superior a 10 conceitos, no intervalo entre 11 a 15 conceitos, e nenhum deles representou em seu primeiro mapa, um número superior a 15 conceitos.

Pode-se observar, que, em geral, a maioria dos alunos apresentou um baixo conhecimento prévio em relação aos conteúdos de genética aqui trabalhados, quando se considera o baixo número de conceitos que foram representados neste primeiro mapa, quando comparados aos mapas subsequentes.

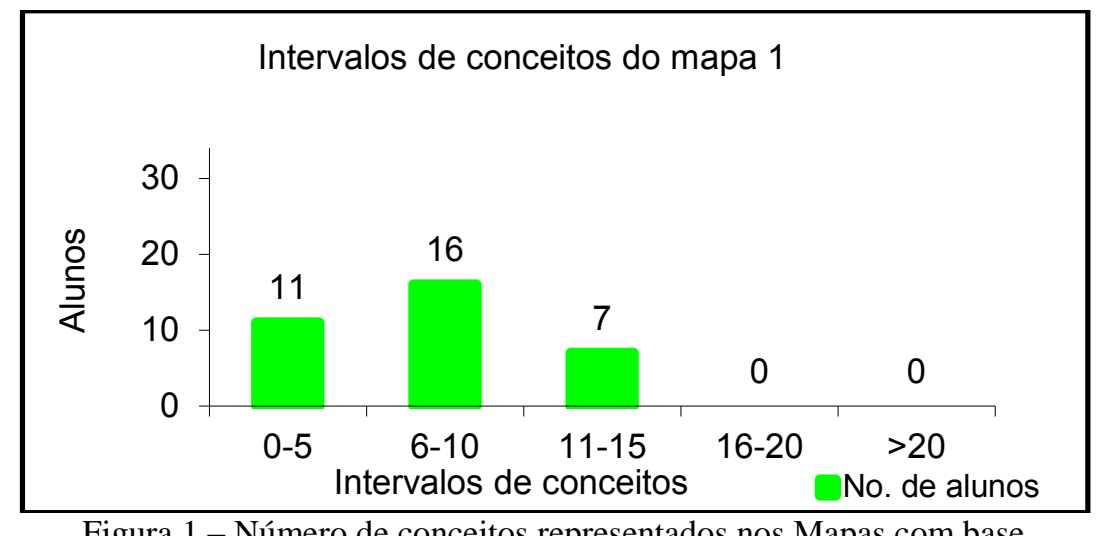

Figura 1 - Número de conceitos representados nos Mapas com base apenas nos conhecimentos prévios dos alunos

Na Figura 2 apresentamos os dados relativos à quantidade de conceitos representados pelos alunos por intervalos. Observamos no segundo mapa elaborado a partir da interação dos alunos com os variados recursos visitados e avaliados pela internet por meio do tablet, apenas dois alunos não representaram um número de conceitos adequados superior a 10. Todos os demais, quase totalidade da amostra pesquisada, conseguiu representar mais de 10 conceitos adequados. Destes, observou-se que 13 alunos representaram acima de 15 conceitos adequados.

Esses resultados da análise do segundo mapa que indicam um número superior de conceitos adequados quando comparados ao primeiro mapa, estão de acordo com as declarações de $\mathrm{O}^{`}$ Nuallain \& Brennan (2004) quando afirmam que dispositivos móveis quando disponibilizam material em diferentes formatos podem reforçar a aprendizagem, e de Seabra (2012) quando reitera que a possibilidade de acesso à informação por diversos formatos como infográficos, simulações, jogos educacionais, enciclopédias digitais de acordo com a preferência e aptidão dos alunos podem favorecer a aprendizagem dos conteúdos. Além disso, acredita-se também que, conforme defende Caceffo, Rocha e Azevedo (2011), a postura ativa de buscar pelo conhecimento pode favorecer um maior engajamento nas atividades propostas e, com isso, favorecer a sua aprendizagem, como foi o observado neste estudo.

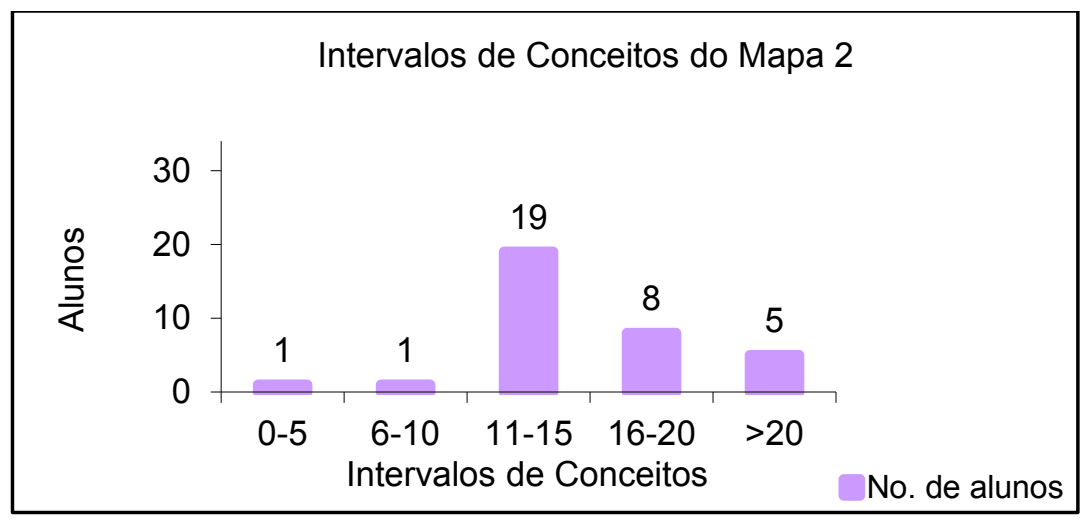


Figura 2 - Número de conceitos de conteúdos de genética representados nos Mapas com base na interação dos alunos com os recursos pesquisados via tablet

Conforme a Figura 3, na terceira versão do mapa digital, elaborada a partir das observações das professoras e colegas durante a explicação dos alunos do segundo mapa, e a partir do feedback recebido das professoras por meio da análise dos mapas disponibilizado no Blackboard, observou-se ainda que apenas um dos alunos representou até dez conceitos em seu mapa. Todo o restante dos alunos representou um número superior a dez conceitos, sendo que 29 alunos, a grande maioria destes, representaram um número superior a 15 conceitos. Esses dados, quando analisados comparativamente aos apresentados no Mapa 1 (Figura 2) elaborado apenas com base no conhecimento prévio dos alunos indicam a ocorrência de uma evolução conceitual da amostra.

Nessa versão do mapa, foi considerada a mediação através dos elementos sociais de mediação (ENGESTRÖM, 1987), como foi o caso das professoras e os demais alunos envolvidos no estudo. $\mathrm{O}$ fato de terem sido criados espaços para que o aluno se manifestasse, emitisse suas opiniões, ouvisse seus colegas e professoras, pode ter propiciado condições de envolvimento com o novo conhecimento a ser estruturado (PERRENOUD, 2000). Para Rodrigues \& Sforni (2010), é na troca com o professor e com os seus pares que os sujeitos vão internalizando conhecimentos, papéis e funções sociais, o que possibilita o desenvolvimento do conhecimento e da consciência. Esse movimento de formação, segundo os autores, caminha do plano social para o plano individual, ou seja, das relações interpsíquicas para as relações intrapsíquicas.

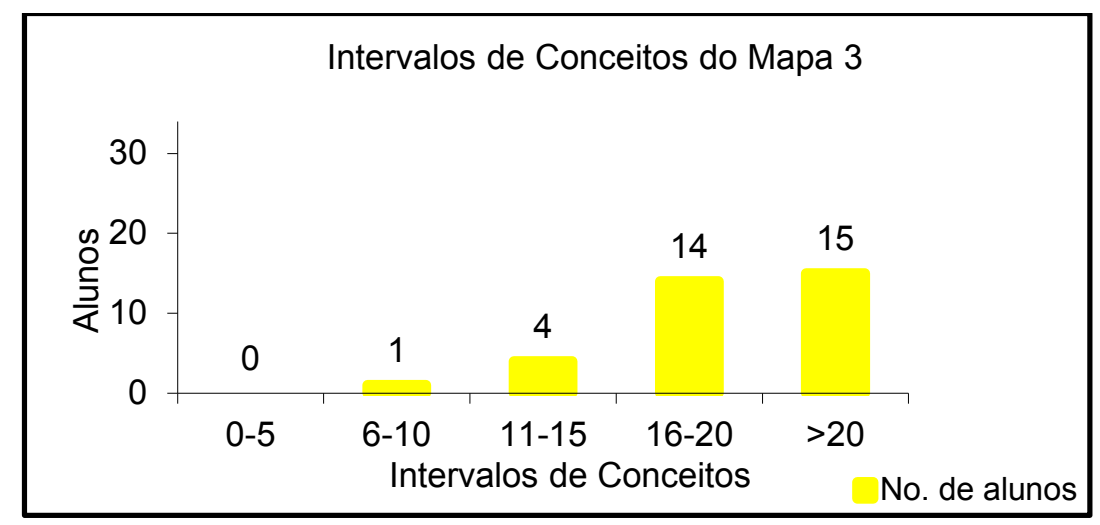

Figura 3 - Número de conceitos de conteúdos de genética representados nos Mapas com base na mediação dos colegas e professoras

A evolução dos conceitos representados pelos estudantes no desenvolvimento da atividade, registrados e analisados pelas professoras participantes da pesquisa, pode ser considerado um indicador da relevância do uso dos tablets na educação e na formação de competência para transformar dados em conhecimento relevante, considerado uma das bases da literacia digital e informacional (RAMOS e FARIA, 2012). Os diversos recursos e potenciais dos tablets e outros dispositivos móveis possibilitam novas e variadas estratégias de ensino e aprendizagem na direção do que se tem chamado de sala de aula invertida ou Flipped Classroom (HERREID e SCHILLER, 2013) e, portanto, uma transformação do espaço e tempo na realização das atividades de ensino e aprendizagem.

\section{CONSIDERAÇÕES FINAIS}


Com base nos resultados apresentados em que se identificou uma evolução no número de conceitos representados pelos alunos ao longo das intervenções realizadas, observa-se que o tablet, quando utilizado, fundamentado em uma teoria adequada como a TA, e com o apoio de uma estratégia apropriada como o mapeamento mental, pode colaborar no processo de ensino e aprendizagem de Biologia na aquisição da competência de representação de conhecimentos de genética sob a forma de conceitos.

A atividade de representação do conhecimento, como foi desenvolvida neste estudo, demanda a realização de ações complexas, como busca por conhecimento na internet, seguida de mapeamento mental com base nos conceitos encontrados. Para isso, deve ser intencionalmente organizada para desenvolver ações e operações mentais qualitativamente superiores (SFORNI, 2004).

A interação dos alunos entre si e com as professoras durante a atividade realizada, seja pessoalmente ou através de espaços de discussão como o Fórum de discussões do Blackboard, constituíram-se operações diferenciadas que permitiram a execução das ações propostas e reforça a importância dos mediadores sociais (ENGESTRÖM, 1987) em situações de ensino e aprendizagem que envolvem o uso de tecnologias.

Este estudo mostrou que é possível proporcionar ao aluno a representação do seu conhecimento por meio do uso do tablet, permitindo-lhe buscar esse conhecimento nas fontes e recursos de seu interesse, necessidade e maior poder de assimilação. Concluímos indicando que a relação ensino e aprendizagem pode ser alterada pelas inovações trazidas por esses dispositivos móveis e esta proposta constituise em uma alternativa de como incorporar o tablet dentro de uma sala de aula por meio da participação ativa do aluno na representação do seu próprio conhecimento.

"O presente trabalho foi realizado com o apoio da CAPES (Coordenação de Aperfeiçoamento de Pessoal de Nível Superior - Brasil”.

\section{Referências}

ALMEIDA, R. R.; ARAUJO JR., C. F. A Teoria da Atividade e sua relação com o uso de dispositivos móveis no ensino. In: ARAUJO JR., C. F.; SILVEIRA, I. F. (Org.). Tablets no Ensino Fundamental e Médio: princípios e aplicações. São Paulo: Terracota, 2012.

ARAÚJO JR, C.F. e SILVEIRA, I.F. Tablets no Ensino Fundamental e Médio: princípios e aplicações. São Paulo: Editora Terracota, 2012.

BASSO, I. S. Significado e sentido do trabalho docente. Cadernos CEDES, Campinas, v. 19, n. 44, p. 19-32, Abr. 1998.

BRASIL. Ministério da Educação, Secretaria da Educação Média e Tecnológica. PCN

Ensino Médio: orientações complementares aos Parâmetros Curriculares Nacionais. Ciências da Natureza, Matemática e suas Tecnologias. Brasília: MEC, SEMTEC, 2002.

BUZAN, T. Mapas mentais e sua elaboração. São Paulo: Editora Cultrix, 2005.

CACEFFO, R. E.; ROCHA, H. V.; AZEVEDO, R. J. Ferramenta de apoio para o aprendizado ativo usando dispositivos com caneta eletrônica. Revista Brasileira de Informática na Educação, Campinas, v.19, n. 2, p. 25-41, Ago. 2011. Disponível em:<http://www.br-ie.org/pub/index.php/rbie/article/view/1267/1174. Acesso em 13 abr. 2013.

CROMPTON, H. Mobile learning: new approach, new theory. In: BERGE, Z. L.; 
MUILENBURG, L. Y. (Ed.). Handbook of mobile learning. New York: Taylor \& Francis, 2013.

ENGESTRÖM, Y. Learning by Expanding: An Activity-Theoretical Approac to Developmental Research. Helsinki: Orienta Konsultit, 1987.

HERREID, Clyde Freeman; SCHILLER, Nancy A. Case studies and the flipped classroom. Journal of College Science Teaching, v. 42, n. 5, p. 62-66, 2013.

HIGUCHI, A. A. S. Tecnologias móveis na educação: Um estudo de caso em uma escola da rede pública do estado de São Paulo. 2011. 92 f. Dissertação de Mestrado. (Educação, Arte e História da Cultura) - Universidade Presbiteriana Mackenzie, São Paulo - SP, 2011.

KAPTELININ, V.; NARDI, B.A. Activity Theory: basic concepts and applications. CHI 97 Electronic Publications: Tutorials. [S.1.] [1997?]. Disponível em: < http://old.sigchi.org/chi97/proceedings/tutorial/bn.htm>. Acesso em: 15 abr. 2013.

LEONTIEV, A. N. Activity, consciousness and personality. Englewood Cliffs: Prentice-Hall, 1978. Disponível em:<http://www.marxists.org/archive/leontev/ works/1977/leon1977.htm >. Acesso em: 15 nov. 2013.

LEONTIEV, A. N. Os princípios psicológicos da brincadeira pré-escolar. In: Vygotsky et al. Linguagem, desenvolvimento e aprendizagem. São Paulo: Ícone, 1988. p.119-142.

OLIVEIRA, J. B. et al. O uso de tablets e o geogebra como ferramentas auxiliadoras no ensino da matemática. Actas de la Conferencia Latinoamerica na Geogebra, v. 1, p. 405-413, 2012.

O’NUALLAIN, C.; BRENNAN, A. How can one effectively assess students working in a collaborative mobile environment on an individual basis? In: ATTEWELL, J.; SAVILL- SMITH, C. (Eds). Mobile Learning Anytime Everywhere, A Book of papers from m-Learn. Londres: LSDA, 2004, p. 149-152.

PERRENOUD, P. Dez novas competências para ensinar. Porto Alegre: Artes Médicas, 2000.

RAMOS, A.; FARIA, P. LITERACIA DIGITAL E LITERACIA INFORMACIONAL: breve análise dos conceitos a partir de uma revisão sistemática de literatura. Linhas, v. 13, n. 2, p. 29-50, 2012.

RODRIGUES, V. L. G. C.; SFORNI, M. S. F. Análise da apropriação do conceito de volume sob a perspectiva da Teoria da Atividade. Ciência e Educação. UNESP, São Paulo, v. 16, p. 543-556, 2010.

SEABRA, C. Tablets na sala de aula. [S.1.] [2012?] Disponível em: < https://cseabra.wordpress.com/2012/04/22/tablets-na-sala-de-aula/ > Acesso em: 12 fev. 2014.

SFORNI, M. S. F. Aprendizagem conceitual e organização do ensino: contribuições da teoria da atividade. Araraquara: JM Editora, 2004.

SHARPLES, M.; TAYLOR, J.; VAVOULA, G. Towards a theory of mobile learning. In: Proceedings of mLearn, v. 1, n. 1, p. 1-9, 2005. Disponível em:

$<$ http://www.mlearn.org/mlearn2005/CD/papers/Sharples\%20Theory\%20of\%20Mobile. pdf $>$. Acesso em: 13 mar. 2013.

VIEIRA, M. C. ; SANTAROSA, L. M. C. Tendências na inserção de dispositivos móveis na educação: uma revisão de literatura internacional. In: XVIII CONFERÊNCIA INTERNACIONAL SOBRE INFORMÁTICA NA EDUCAÇÃO, 2013, Porto Alegre. Nuevas Ideas en Informática Educativa: Memórias del XVIII Conferência Internacional de Informática Educativa, TISE, 2013. v. 9. p. 495-498.

VYGOTSKY, L. S. Mind in Society - The Development of Higher Psycological

Process. Cambridge MA: Harvard University Press, 1978. 
